\title{
DERECHO Y POLÍTICAS AMBIENTALES EN GALICIA (SEGUNDO SEMESTRE 2016)
}

\author{
ANDREA GARRIDO JUNCAL \\ Profesora contratada interina de Derecho Administrativo \\ Universidad de Santiago de Compostela
}

Sumario: 1. El continuismo de los gobiernos que dirigen la Administración autonómica gallega y la Administración estatal. 2. ENCE: los dos últimos pronunciamientos del Tribunal Supremo y el Pacto Ambiental suscrito por la entidad mercantil con la Xunta de Galicia. 3. Reganosa: la exclusión del trámite de evaluación de impacto ambiental acordada por el Consejo de Ministros y la anulación judicial del Plan de Emergencia. 4. La aprobación del Reglamento que desarrolla la Ley 2/2016, de 10 de febrero, del suelo de Galicia. 5. Decretos que inciden en la materia ambiental. 5.1. La creación de la Red de Parques Naturales de Galicia. 5.2. El entierro de las mascotas y otros animales. 5.3. El Catálogo de los Paisajes de Galicia. 5.4. El nuevo Plan Territorial de Contingencias por Contaminación Marina Accidental.

\section{El continuismo de los gobiernos que dirigen la Administración autonómica gallega y la Administración estatal}

El 2 de agosto de 2016 se publicó en el Diario Oficial de Galicia (DOG) el Decreto 92/2016, de 1 de agosto, de disolución del Parlamento de Galicia y de convocatoria de elecciones. El 25 de septiembre de este año el Partido Popular lograba 41 de los 75 escaños en disputa, de forma que Alberto Núñez Feijoo conserva la mayoría absoluta en la Cámara gallega. El recién investido presidente de la Xunta de Galicia seguirá gobernando los próximos cuatro años sin incluir, en principio, ningún cambio en el equipo que dirige la Administración autonómica. Como consecuencia de la decisión de mantener intacta la estructura orgánica del Gobierno de Galicia, Beatriz Mato Otero asumía de nuevo, el 13 de noviembre de 2016, el cargo de Conselleira de Medio Ambiente y Ordenación del Territorio.

$\mathrm{Al}$ examinar los resultados de esta cita electoral, resulta pertinente reparar en el empate, en número de diputados pero no en número de votos, entre En Marea y el Partido Socialista. La coalición de Anova, En Marea, Podemos y Esquerda Unida ha conseguido posicionarse como la segunda fuerza política más votada tras la debacle de los socialistas en nuestra comunidad autónoma, quienes han obtenido el peor resultado de su historia y 
han perdido cuatro escaños con respecto al 2012. Por su parte, el Bloque Nacionalista Galego ha obtenido seis escaños y Ciudadanos se ha quedado sin representación parlamentaria.

En el contexto político nacional, el 29 de octubre de 2016 el candidato a la Presidencia del Gobierno, Mariano Rajoy Brey, se sometía a la segunda votación de investidura al no haber obtenido la mayoría absoluta que exige la Constitución. Con 170 votos afirmativos, 111 negativos y 68 abstenciones, se ponía fin al Gobierno en funciones y cinco días más tarde los ministros juraban su cargo ante el rey Felipe VI en el Palacio de la Zarzuela de Madrid. Al frente del Ministerio de Agricultura y Pesca, Alimentación y Medio Ambiente repite Isabel García Tejerina. Al igual que Alberto Núñez Feijoo, Mariano Rajoy Brey ha optado por el continuismo de su gabinete, salvo en el caso de José Manuel GarcíaMargallo y Jorge Fernández Díaz.

\section{ENCE: los dos últimos pronunciamientos del Tribunal Supremo y el pacto ambiental suscrito por la entidad mercantil con la Xunta de Galicia}

El 21 de junio de 2016 la Sección Quinta de la Sala de lo Contencioso-Administrativo del Tribunal Supremo sentenció que no procedía el recurso de casación interpuesto por el Ayuntamiento de Pontevedra contra los autos dictados, con fechas 10 de abril y 3 de junio de 2015, por la Sección Primera de la Sala de lo Contencioso-Administrativo del Tribunal Superior de Justicia de Galicia, en los que se desestimaba el incidente de ejecución de la sentencia pronunciada por dicha Sala, con fecha 2 de octubre de 2012, en el procedimiento ordinario 149 de 2004.

A juicio del Alto Tribunal, la corporación municipal parte de una premisa errónea como es la anulación en vía judicial del Proyecto Sectorial de Incidencia Supramunicipal aprobado por Decreto del Consejo de Gobierno de la Xunta de Galicia de 26 de diciembre de 2003, cuando lo único que se anuló fueron las determinaciones relativas a una fábrica de papel tisú debido a la carencia de una serie de informes sectoriales.

Teniendo en cuenta la farragosa sentencia cuya ejecución fue requerida por el ente local, el Tribunal Supremo decidió que concurrían las circunstancias que justificaban la no condena al pago de las costas causadas (fundamento de derecho $2 .^{\circ}$ ). En nuestra opinión, el órgano jurisdiccional, además de reconocer que el contenido del pronunciamiento 
judicial podría haber sido redactado con mayor claridad, debería también haber evitado realizar la siguiente apreciación: “[...] se evidencia la obstinación de la Corporación municipal en atribuir a la sentencia, cuya ejecución pidió, un significado y alcance que no tiene" (fundamento de derecho $1 .^{\circ}$ ).

Un día más tarde, la Sección Quinta de la Sala de lo Contencioso-Administrativo del Tribunal Supremo dictó otra sentencia en la que se declaraba que el único motivo de casación alegado debía ser desestimado. Como se ha señalado con anterioridad, el Proyecto Sectorial de Incidencia Supramunicipal no fue anulado y sus determinaciones fueron publicadas en el DOG el 14 de diciembre de 2012, de modo que, al ser válidas y eficaces, condicionan el planeamiento urbanístico municipal y, dado que aquel contiene la regulación detallada del suelo ocupado por las instalaciones, tal regulación prevalece sobre el planeamiento municipal por disposición expresa de la Ley 1/1997, de 24 de marzo, del Suelo de Galicia, por lo que no cabe admitir, como pretende el Ayuntamiento de Pontevedra, que la autorización ambiental integrada no sea compatible urbanísticamente con dicho planeamiento, ya que este debe ajustarse a la regulación detallada del suelo contenida en el Proyecto Sectorial (fundamento de derecho $4 .^{\circ}$ ).

Inmediatamente después del conocimiento de estos fallos, la Xunta de Galicia firmó un pacto ambiental con ENCE por el que la empresa se compromete a llevar a cabo inversiones en materia económica, social y medioambiental. El documento firmado constituye una hoja de ruta que incluye iniciativas concretas, con los presupuestos consignados para cada una de ellas y con sus respectivos calendarios de ejecución. Entre otras, cabe destacar que se destinarán doce millones de euros a la reducción del impacto acústico y de olores, unos quince millones al tratamiento y la regeneración de aguas en el complejo, y cerca de cuatro millones a la integración paisajística. Asimismo, se prevé la puesta en marcha de un programa marco para programas sociales y ambientales con la consignación de tres millones de euros anuales, que permitirá la concesión de bolsas y ayudas. Se asume además el compromiso de poner en funcionamiento un centro de investigación especializado en la explotación sostenible de los recursos forestales, para lo cual se propone el Pazo de Lourizán como sede, y se proyecta igualmente la construcción y puesta en marcha de un nuevo centro de cogeneración de biomasa en las 
instalaciones de Lourizán, así como de tres nuevos centros de bioenergía en distintos puntos de Galicia ${ }^{1}$.

\section{Reganosa: la exclusión del trámite de evaluación de impacto ambiental acordada por el Consejo de Ministros y la anulación judicial del Plan de Emergencia}

El 27 de mayo de 2016 el Consejo de Ministros acordó excluir, de manera excepcional, del trámite de evaluación de impacto ambiental los proyectos de autorización de la planta de recepción, almacenamiento y regasificación de gas natural licuado emplazada en el municipio de Mugardos (A Coruña) y propiedad de la entidad mercantil Regasificadora del Noroeste, S. A. (Reganosa).

Desde el punto de vista de este órgano colegiado del Gobierno, esta medida evita una paralización prolongada de la actividad de las instalaciones del referido establecimiento industrial que pondría en riesgo el suministro de gas natural en España y Portugal, así como la seguridad de personas, de bienes y del propio sistema gasista. Utilizando sus propias palabras, "se pretende minimizar los perjuicios que provocaría su paralización a los consumidores de la zona noroeste, al sistema eléctrico Español (por la indisponibilidad de los ciclos combinados a los que se suministra gas desde la planta) e, incluso, al sistema gasista portugués". A mayor abundamiento, se resalta que varios informes - el del gestor técnico de Enagás y los de las direcciones generales de Tráfico, de Protección Civil y Emergencias y de Transporte Terrestre- constatan la concurrencia de circunstancias excepcionales en el presente caso y que, de todas formas, se ha realizado una evaluación que concluye que la planta "es viable ambientalmente" y que "no tiene ninguna incidencia significativa susceptible de producir repercusiones apreciables en el medio ambiente o los recursos naturales", sin perjuicio de la posible imposición en un futuro de la obligación del establecimiento de medidas adicionales de prevención, corrección y vigilancia como condición de operación de la planta.

La Sección Quinta de la Sala de lo Contencioso-Administrativo del Tribunal Supremo dictó sentencia, con fecha 26 de julio de 2016, en la que se anunciaba que el Decreto 144/2007, de 19 de julio, de la Xunta de Galicia, por el que se aprueba el Plan de Emergencia Exterior de la planta de recepción, almacenamiento, regasificación y

\footnotetext{
${ }^{1}$ Sobre el compromiso de inversión de 200 millones de euros en los próximos cuatro años, véase la nota de prensa disponible en http://www.xunta.gal/notas-de-prensa/-/nova/2107/xunta-asina-pacto-ambiental-conence-que-recolle-compromisos-ambientais-sociais
} 
expedición de gas licuado de Reganosa, era contrario a derecho y, en consecuencia, debía ser anulado conforme a los artículos 63.1 de la Ley 30/1992, de 26 de noviembre, de Régimen Jurídico de las Administraciones Públicas y del Procedimiento Administrativo Común, y 68.1.b), 70.2, 71.1.a) y 72.2 de la Ley 29/1998, de 13 de julio, reguladora de la Jurisdicción Contencioso-Administrativa. En el antecedente de hecho sexto se precisa que el recurso de casación interpuesto por la Plataforma de Vecinos O Cruceiro de Mehá se basa en cuatro motivos que son el resultado de la infracción de los artículos 12, 11, 9, 8 y 4 del Real Decreto 1254/1999, de 16 julio, por el que se aprueban medidas de control de los riesgos inherentes a los accidentes graves en los que intervengan sustancias peligrosas.

En primer lugar, desde la perspectiva de la parte recurrente, el estudio de las distancias medias y de los posibles riesgos inherentes a la planta no se ha realizado de acuerdo con un plan urbanístico válido, por lo que carece de sentido que no se considere relevante que el Plan General de Ordenación Urbana, que permitió la ubicación de la planta, haya sido declarado nulo en sentencia firme del Tribunal Supremo por no disponer de la preceptiva evaluación ambiental, en la que se tendría que haber realizado un análisis de los riesgos y efectos que para la seguridad y salud de las personas podría implicar la implantación de dicha planta. En segundo lugar, se denuncia una vulneración del procedimiento legalmente establecido respecto a la notificación, elaboración del informe de seguridad y del propio Plan de Emergencia Exterior, así como respecto a la obligación de establecer mecanismos de consulta a la población, llegando a construirse las instalaciones y a acordar la puesta en servicio parcial y definitiva cuando tanto la autorización previa como la autorización de construcción estaban recurridas en casación, habiéndose iniciado el funcionamiento de la instalación sin el informe de seguridad que se debe presentar con carácter previo a la construcción conforme al artículo 9 del Real Decreto 1254/1999 y sin haberse establecido mecanismos de consulta a la población, a la que se le ocultaron los verdaderos riesgos de la planta de Reganosa puesto que se le dijo que el gas natural no arde ni explota, mientras que los folletos de información sobre las normas a seguir en situaciones de riesgo — que la Xunta de Galicia remitió a los ciudadanos que viven en las proximidades de Punta Promontorio en noviembre de 2008 - señalaron el riesgo de que se produzca una explosión, incendio o fuga y las consecuencias gravísimas de ello. En tercer lugar, se manifiesta que la previsión del efecto dominó en el Plan de Emergencia Exterior es deficiente debido a la ubicación y proximidad de instalaciones en las que se 
hallan sustancias peligrosas, al haber contado meramente con un simple estudio de dos páginas elaborado cinco años atrás para Forestal del Atlántico. En cuarto y último lugar, se apunta a una conculcación del artículo 4 del Real Decreto 1254/1999 al excluir de su ámbito de actuación las operaciones de carga y descarga de gas natural licuado (GNL) por parte de los buques metaneros.

Antes de entrar en el examen de los motivos de casación alegados, la Sección Quinta de la Sala de lo Contencioso-Administrativo del Tribunal Supremo explica que la representación procesal de la Administración de la Comunidad Autónoma de Galicia, comparecida como parte recurrida, sostiene que la asociación recurrente trae a debate cuestiones de manera indebida al omitir los preceptos en los que sus pretensiones se amparan. La referida oposición al recurso de casación no es admitida por el órgano jurisdiccional, quien asegura que en el escrito de interposición del recurso se esgrime, sin lugar a duda, la infracción de diferentes disposiciones.

Sin la realización de ningún comentario remarcable acerca de las alegaciones formuladas por la entidad mercantil Reganosa, la otra parte recurrida, se extrae la conclusión de que no es aceptable el argumento de que el artículo 12 del Real Decreto 1254/1999 se proyecta sobre los instrumentos urbanísticos y de ordenación del territorio, cuya aplicación corresponde a las administraciones autonómica o municipal urbanísticas, pero que no es aplicable cuando se trata de aprobar un plan de emergencia. La Sección Quinta de la Sala de lo Contencioso-Administrativo del Tribunal Supremo estima el primero de los motivos de casación, con la consiguiente anulación de la sentencia pronunciada, con fecha 20 de marzo de 2014, por la Sección Segunda de la Sala de lo Contencioso-Administrativo del Tribunal Superior de Justicia de Galicia en el recurso contencioso-administrativo número 4290 de 2008.

La aceptación del primer motivo de casación hace innecesario examinar el resto dado que, aun cuando en el Plan de Emergencia aprobado por el Decreto autonómico impugnado se hayan observado las reglas de procedimiento recogidas en el Real Decreto 1254/1999, lo cierto es que falta el presupuesto de que la planta o instalación para la que se elabora dicho Plan disponga de cobertura urbanística. Además, se añade que la autorización administrativa previa para dicha instalación y su proyecto de ejecución han sido anulados por sentencias de la Sala Tercera del Tribunal Supremo de fechas 28 de marzo de 2016 (recurso de casación 599/2013) y 25 de abril de 2016 (recurso de casación 2175/2013) y que, consecuentemente, no es acorde con el ordenamiento jurídico aplicable 
la aprobación de un plan de emergencia exterior para la instalación de una planta cuya autorización administrativa previa ha sido anulada, al igual que el proyecto de ejecución de dicha instalación.

A partir del argumento precedente, la representación procesal de Reganosa señala que el 9 de julio de 2016 se publicó en el Boletín Oficial del Estado la Resolución de 7 de julio de 2016, de la Dirección General de Política Energética y Minas, por la que se otorgaba a Reganosa la autorización administrativa y se aprobaba el proyecto de ejecución de las instalaciones de planta de recepción, almacenamiento y regasificación de gas natural licuado en Mugardos. Sin embargo, la Sección Quinta de la Sala de lo ContenciosoAdministrativo del Tribunal Supremo puntualiza que su decisión no supone ninguna contradicción, ya que el debido Plan de Emergencia Exterior para esa planta deberá aprobarse en atención a su emplazamiento urbanístico de acuerdo con el Plan General de Ordenación Urbana Municipal y a esa autorización previa y proyecto de ejecución con los que, al parecer, cuenta ahora la referida entidad mercantil titular de la planta en cuestión.

\section{La aprobación del Reglamento que desarrolla la Ley 2/2016, de 10 de febrero, del Suelo de Galicia}

El 9 de noviembre se publicó en el DOG el Decreto 143/2016, de 22 de septiembre, que aprueba el Reglamento de la Ley 2/2016, de 10 de febrero, del Suelo de Galicia ${ }^{2}$. El objetivo es completar, actualizar y facilitar la aplicación de la legislación urbanística. Con el fin de permitir el manejo unitario de todo el cuerpo normativo, se elabora un texto que, además de constituir el desarrollo reglamentario propiamente dicho, incorpora la regulación existente en la propia ley.

En aras de la coherencia interna de toda la materia urbanística, el Reglamento incorpora todas aquellas cuestiones que hasta ahora estaban contenidas en las siguientes normas: Decreto 28/1999, de 21 de enero, por el que se aprueba el Reglamento de Disciplina Urbanística para el desarrollo y aplicación de la Ley 1/1997, de 24 de marzo, del Suelo de Galicia; Reglamento de Planeamiento Urbanístico, aprobado por el Real Decreto

\footnotetext{
${ }^{2}$ Para un conocimiento de las principales novedades que supone la entrada en vigor de la Ley 2/2016, de 10 de febrero, del Suelo de Galicia, se recomienda la lectura de la crónica escrita por A. NOGUEIRA LÓPEZ en la Revista Catalana de Dret Ambiental, núm. 1, 2016.
} 
2159/1978, de 23 de junio; Reglamento de Disciplina Urbanística, aprobado por el Real Decreto 2187/1978, de 23 de junio; y Reglamento de Gestión Urbanística, aprobado por el Real Decreto 3288/1978, de 25 de agosto. Lo indicado anteriormente supone la derogación, o inaplicación, de la normativa reseñada ${ }^{3}$.

La estructura del Reglamento resulta similar a la de la Ley 2/2016, de 10 de febrero, del Suelo de Galicia, dividiéndose en siete títulos. A continuación nos limitaremos a apuntar sucintamente los temas que han sido merecedores de una pormenorización regulatoria.

El título preliminar recoge los principios generales de la actividad urbanística y define las competencias de cada una de las administraciones implicadas, así como de algunos de sus órganos y de entidades a ellas adscritas como la Comisión Superior de Urbanismo, la Agencia de Protección de la Legalidad Urbanística o el Jurado de Expropiación de Galicia ${ }^{4}$.

El título I realiza precisiones puntuales acerca de la clasificación y categorización del suelo, así como del régimen jurídico correspondiente en función de estas. En cuanto al régimen del suelo urbano, se mantienen en lo esencial los criterios tradicionales para su clasificación y categorización, diferenciando entre el suelo urbano consolidado y no consolidado, adaptando las definiciones de estas categorías de suelo a los conceptos contenidos en la legislación básica estatal del suelo, y clarificando la definición de las actuaciones que implican procesos de urbanización, las de reforma y renovación y las de dotación. Respecto del suelo de núcleo rural, categorizado como tradicional y común, el Reglamento desarrolla los criterios para la delimitación de los núcleos rurales y los métodos para el cálculo del grado de consolidación que se debe cumplir en ellos. En relación con el suelo urbanizable, se apuesta por abandonar el carácter residual que tradicionalmente se le venía atribuyendo, y, en este sentido, se define como el relativo a los terrenos que el planeamiento considere necesarios y acomodados para permitir el crecimiento de la población y de la actividad económica o para completar su estructura urbanística. La regulación del suelo rústico, con las categorías de suelo rústico de protección ordinaria y de especial protección, no difiere de lo indicado en la Ley, pero se

\footnotetext{
${ }^{3}$ Véanse la disposición derogatoria única y la disposición final segunda del Decreto 143/2016, de 22 de septiembre, que aprueba el Reglamento de la Ley 2/2016, de 10 de febrero, del Suelo de Galicia.

${ }^{4}$ Adviértase que se modifican los números 1, 2, 3 y 4 del artículo 4 del Decreto 223/2005, de 16 de junio, por el que se aprueba el Reglamento de organización y funcionamiento del Jurado de Expropiación de Galicia.
} 
introducen precisiones que derivan de la necesidad de tener en cuenta lo previsto en la legislación básica estatal. Al mismo tiempo, se incluye el concepto de reestructuración parcelaria, que viene a substituir al de concentración parcelaria. En cuanto a los usos cuya implantación se considera que debe ser objeto de una mayor tutela por parte de la Administración de la Comunidad Autónoma (las construcciones destinadas a usos residenciales vinculados a las explotaciones agropecuarias y las construcciones que alberguen actividades complementarias de primera transformación de productos del sector primario), el Reglamento especifica los requisitos y las condiciones que deben concurrir para que se pueda otorgar la autorización autonómica a ese tipo de usos.

El título II se dedica a la regulación del planeamiento urbanístico. En primer lugar, se incluyen en esta parte del Reglamento una serie de disposiciones generales para todos los instrumentos de planeamiento urbanístico y se acomete el esfuerzo de delimitar con nitidez lo que debe entenderse por dotaciones urbanísticas. En un segundo término, se detallan las determinaciones, el contenido de la documentación y el proceso de tramitación de cada uno de los instrumentos de planeamiento urbanístico previstos en la Ley 2/2016, de 10 de febrero, del Suelo de Galicia, poniendo una mayor atención en la figura del plan general de ordenación municipal, pues es el instrumento de planeamiento de mayor complejidad y el principal para el desarrollo de la mayor parte de los ayuntamientos de Galicia.

El título III regula las normas de aplicación directa relativas a la adaptación al ambiente y a la protección del paisaje y de las vías de circulación. Estas normas suponen un mecanismo para preservar el medio ambiente y el patrimonio natural y cultural y sirven, más concretamente, como principios que tienen que regir la ordenación de un territorio en ausencia de planeamiento.

El título IV se centra en la ejecución del planeamiento urbanístico. Con la voluntad de dotar de una mayor seguridad jurídica la gestión urbanística, se precisan aspectos capitales de las obras de urbanización y se introduce la novedad de habilitar a los ayuntamientos para aprobar, en casos motivados, proyectos de obras en actuaciones sistemáticas con el fin público de ejecutar parcelas dotacionales públicas. Se señala que, acomodándose sustancialmente a la Ley 2/2016, de 10 de febrero, del Suelo de Galicia, se lleva a cabo un reajuste en la delimitación de las áreas de reparto, aprovechamiento tipo y polígonos de ejecución. Partiendo de la complejidad de la equidistribución, el Reglamento pone de relieve la conveniencia de garantizar el correcto desarrollo de las 
actuaciones reparcelatorias. En esta línea, con el objeto distribuir justamente las cargas y los beneficios de la ordenación urbanística, regularizar la configuración de las fincas, situar su aprovechamiento en zonas aptas para la edificación de acuerdo con el planeamiento y localizar sobre parcelas determinadas el aprovechamiento, se especifican los órganos que están legitimados para la formulación del proyecto de equidistribución, se precisa el contenido de este proyecto y se enumeran los sujetos que tendrán la consideración de parte en estos expedientes. Las previsiones reglamentarias serán de aplicación obligatoria a los instrumentos de equidistribución que se formulen bajo los sistemas de cooperación, concierto y compensación. Se abordan también los sistemas de actuación, desarrollando la facultad de proceder a su modificación y regulando aspectos propios de los sistemas como las formas de colaboración por las personas propietarias en el sistema de cooperación, el contenido del proyecto de estatutos y de bases de actuación o la constitución de la junta de compensación en el sistema de compensación.

En consonancia con el Real Decreto Legislativo 7/2015, de 30 de octubre, por el que se aprueba el texto refundido de la Ley de Suelo y Rehabilitación Urbana, el título V describe minuciosamente los patrimonios públicos del suelo como un mecanismo de intervención en el mercado del suelo.

El título VI aborda la intervención en la edificación y uso del suelo, así como la disciplina urbanística, a través de tres capítulos dedicados al fomento de la edificación, conservación y rehabilitación, a la intervención en la edificación y uso del suelo, y a la disciplina urbanística. Se subraya el deber de las personas propietarias de finalizar las edificaciones para cuya ejecución obtuvieron la preceptiva licencia, englobándose dentro del deber genérico de edificar los solares. En relación con la intervención en la edificación y uso del suelo, la comunicación previa se consolida como el régimen general de intervención administrativa, manteniéndose la exigencia de licencia urbanística para los actos expresamente relacionados en la propia ley. Nótese que el 9 de noviembre de 2016 se publicó en el DOG — además del Decreto 143/2016, de 22 de septiembre, que aprueba el Reglamento de la Ley 2/2016, de 10 de febrero, del Suelo de Galicia- el Decreto 144/2016, de 22 de septiembre, por el que se aprueba el Reglamento único de regulación integrada de actividades económicas y apertura de establecimientos. La segunda norma de carácter reglamentario representa un paso más en la generalización de la comunicación previa en nuestra comunidad autónoma y completa el régimen jurídico de la evaluación 
de incidencia ambiental, instaurado en la Ley 9/2013, de 19 de diciembre, del Emprendimiento y de la Competitividad Económica de Galicia ${ }^{5}$.

Finalmente, los convenios urbanísticos son objeto de regulación en el título VII, en el que se desarrollan las líneas genéricas relativas a su formalización y perfeccionamiento contenidas en la Ley 2/2016, de 10 de febrero, del Suelo de Galicia.

\section{Decretos que inciden en la materia ambiental}

\subsection{La creación de la Red de Parques Naturales de Galicia}

La Ley 9/2001, de 21 agosto, de Conservación de la Naturaleza de Galicia, define con carácter general los espacios naturales que han de considerarse merecedores de una protección especial, establece sus categorías, regula su procedimiento de declaración y dispone su régimen general de protección, contemplándose la posibilidad de establecer regímenes de protección preventiva. En función de los bienes y valores a proteger, la Ley distingue nueve categorías de espacios naturales protegidos: reserva natural, parque nacional, parque natural, monumento natural, humedal protegido, paisaje protegido, zona de especial protección de los valores naturales, espacio natural de interés local y espacio privado de interés natural (art. 9). Los parques, que pueden ser naturales o nacionales, son áreas naturales poco transformadas por las actividades humanas que, en razón de la belleza de sus parajes, de la representatividad de sus ecosistemas o de la singularidad de su flora, fauna o formaciones geomorfológicas, poseen unos valores ecológicos, estéticos, educativos o científicos cuya conservación exige una atención preferente. En la actualidad existen en Galicia seis espacios naturales que ostentan la calificación de parque natural: el monte Aloia, declarado por el Real Decreto 3160, de 4 de diciembre de 1978; la Baixa Limia-Serra do Xurés, declarado por el Decreto 29/1993, de 11 de febrero; el complejo dunar de Corrubedo y lagunas de Carregal y Vixán, declarado por el Decreto 139/1992, de 5 de junio; las Fragas do Eume, declarado por Decreto 218/1997, de 30 de julio; el

\footnotetext{
${ }^{5}$ Véanse los artículos 24 a 31 del Decreto 144/2016, de 22 de septiembre, por el que se aprueba el Reglamento único de regulación integrada de actividades económicas y apertura de establecimientos. Para una aproximación crítica al articulado aplicable en esta materia, nos remitimos a A. GARRIDO JUNCAL, "Las transformaciones del régimen de intervención administrativa en el procedimiento de evaluación de incidencia ambiental de actividades. El supuesto particular de la legislación gallega", Revista Catalana de Dret Ambiental, núm. 1, 2016.
} 
Invernadeiro, declarado por el Decreto 155/1997, de 5 de junio; y el Parque Natural Serra da Enciña da Lastra, declarado por el Decreto 157/2002, de 4 de abril.

Con el propósito de facilitar una gestión coordinada de estos espacios, que representan más de 57.000 hectáreas del territorio gallego y que reciben cada año 650.00 visitantes, el 21 de junio de 2016 se publicó en el DOG el Decreto 69/2016, de 19 de mayo, por el que se crea la Red de Parques Naturales de Galicia. Los objetivos de dicha red son: “a) convertir los parques naturales en un modelo de gestión compartida y coordinada; b) fomentar la cooperación y promover proyectos comunes; c) potenciar los parques naturales como valor de desarrollo económico y dinamizar las economías locales de su entorno; d) conocer los datos y cifras relativas a la gestión de las principales áreas de actuación en la Red de parques naturales de Galicia; e) situar la marca Parques Naturales de Galicia como referente en España bajo una identidad corporativa global; f) capitalizar el amplio y diverso patrimonio natural de Galicia a través de la promoción de los parques naturales; y g) potenciar las singularidades de cada parque natural para convertir la red en icono de la España verde y como espacios naturales de interés en Europa” (art. 2). El cumplimiento de los objetivos transcritos será responsabilidad de un centro directivo al que la Consellería de Medio Ambiente y Ordenación del Territorio de la Xunta de Galicia le atribuirá competencias en materia de conservación de la naturaleza y dependerá también de las personas representantes de las juntas consultivas de los parques naturales, a quienes la Administración autonómica convocará para asistir a reuniones periódicas en las que se tratarán asuntos de interés común (art. 3).

\subsection{El entierro de las mascotas y otros animales}

A causa de diversas alertas alimentarias, principalmente la llamada crisis de las vacas locas, la Unión Europea se dotó de una legislación en materia de seguridad alimentaria basada en evitar el desvío de determinados subproductos de origen animal y productos derivados no destinados a consumo humano (SANDACH) a la cadena alimentaria humana o animal. Las mismas preocupaciones impulsaron en España la aprobación del Real Decreto 1528/2012, de 8 de noviembre, por el que se establecen las normas aplicables a los subproductos animales y los productos derivados no destinados al consumo humano, cuyo artículo 2 dispone que los órganos competentes de las comunidades autónomas o ciudades de Ceuta y Melilla, así como de las entidades locales, 
y los órganos competentes de la Administración general del Estado — en lo que se refiere a los intercambios con terceros países - serán la autoridad facultada para garantizar el cumplimiento de los requisitos del Real Decreto y de la normativa de la Unión Europea en materia de SANDACH. En virtud de esta habilitación y con apoyo de los artículos 30.1.3, 33.1 y 33.4, el 22 de junio de 2016 se publicó en el DOG el Decreto 72/2016, de 9 de junio, por el que se autorizan y se regulan determinados sistemas de eliminación de subproductos animales no destinados al consumo humano en Galicia y se concretan determinados aspectos sanitarios de las explotaciones porcinas.

Un repaso de los veintiún preceptos, de la disposición adicional primera y de las dos disposiciones finales en los que se estructura el Decreto impide vaticinar que el nuevo marco jurídico vaya a contribuir con éxito a la protección de la salud pública, la sanidad animal y el medio ambiente. A título ejemplificativo, el artículo 7 establece que la “eliminación de los cadáveres de animales de compañía podrá realizarse mediante su enterramiento controlado bien por parte de las personas propietarias de ellos o bien por establecimientos que presten un servicio de enterramiento de animales de compañía a terceros, según lo establecido en este decreto". Además, en el caso de animales de la especie canina, "será requisito imprescindible para poder acogerse a esta vía de eliminación el cumplimiento de los requisitos previos de identificación e inclusión en el registro correspondiente establecidos en el Decreto 90/2002, de 28 de febrero, por el que se regula la tenencia de animales potencialmente peligrosos en la Comunidad Autónoma de Galicia y se crean los registros gallegos de Identificación de Animales de Compañía y Potencialmente Peligrosos y de Entrenadores Caninos”. El artículo 11 declara, en el primer apartado, que "las personas propietarias de los animales de compañía podrán enterrar estos en terrenos de su propiedad o en terrenos ajenos, pero respecto de los que se ostente un título que lo permita". A ello se añade la obligación de que los enterramientos se efectúen siguiendo unos "ritos funerarios", pues el artículo 9 prevé: "1. Los enterramientos se realizarán de forma que se evite la contaminación de las capas freáticas y de los acuíferos y, en todo caso, deberán guardar una distancia mínima de 250 metros desde cualquier captación de agua potable y 50 metros desde cualquier curso de agua. 2. Los enterramientos se realizarán de forma que se eviten otros riesgos para la salud pública o la sanidad animal, mismo por los ruidos u olores. 3. Los enterramientos deberán realizarse de forma y con la profundidad que garantice que los animales carroñeros, oportunistas o plagas no puedan acceder a ellos y no se expongan otros riesgos 
añadidos para la salud pública y/o para la sanidad animal. 4. Los cadáveres o subproductos en la fosa, antes de ser enterrados, deberán ser cubiertos o impregnados con un desinfectante apropiado, como puede ser la cal viva, distribuido uniformemente entre capa y capa de subproducto. 5. En los enterramientos se evitarán, asimismo, daños o alteraciones al medio ambiente natural, en concreto, riesgos para el agua, el aire y el suelo, a la biota, elementos geomorfológicos y elementos de significación patrimonial, cultural o histórica".

En caso de incumplimiento de lo dispuesto en este decreto, el artículo 21 indica que será de aplicación, en función de la materia, el régimen de infracciones y sanciones establecido en la Ley 8/2003, de 24 de abril, de Sanidad Animal, en la Ley 17/2011, de 5 de julio, de Seguridad Alimentaria y Nutrición, en la Ley 22/2011, de 28 de julio, de Residuos y Suelos Contaminados, en la Ley 33/2011, de 4 de octubre, General de Salud Pública, en la Ley 50/1999, de 23 de diciembre, sobre el régimen jurídico de la tenencia de animales potencialmente peligrosos, y en la Ley 1/1993, de 13 de abril, de Protección de Animales Domésticos y Salvajes en Cautividad. La práctica extendida de depositar los restos de las mascotas en las propiedades privadas (en los jardines de las casas, por ejemplo), por una parte, y la fragmentación de la normativa sancionadora, por otra, auguran una escasa persecución de las ilegalidades en este campo.

\subsection{El Catálogo de los Paisajes de Galicia}

El 25 de agosto de 2016 se publicó en el DOG el Decreto 119/2016, de 28 de julio, por el que se aprueba el Catálogo de los Paisajes de Galicia. Según el artículo 9 de la Ley 7/2008, de 7 de julio, de Protección del Paisaje de Galicia, los catálogos del paisaje son "los documentos de referencia que fundamentándose en las distintas áreas geográficas, morfológicas, urbanas y litorales existentes en el territorio gallego deberán delimitar, en base a los diferentes estudios y trabajos existentes en la materia, las grandes áreas paisajísticas de Galicia, identificando los diversos tipos de paisajes existentes en cada una de ellas y sus características diferenciales". El contenido de los catálogos del paisaje se detalla en este mismo artículo, que también establece que podrán, en su caso, identificar determinadas zonas geográficas como "áreas de especial interés paisajístico" en atención a los valores naturales y culturales allí presentes, lo que se lleva a cabo en el Decreto 119/2016, de 28 de julio (art. 3). 
Durante la inauguración de la jornada "Diálogos con el paisaje construido", celebrada el 27 de julio de 2016, el presidente del Gobierno gallego destacó las significativas aportaciones que va a conllevar el primer Catálogo de los Paisajes de Galicia, cuyo decreto pone en valor no solo la defensa de los paisajes gallegos, sino también su diversidad y riqueza. No en vano, este texto identifica 258 tipos de paisajes diferentes en la geografía gallega y delimita 211 áreas de especial interés paisajístico que abarcan más de 252.595 hectáreas. El titular del Ejecutivo autonómico se refirió igualmente al documento como un ejemplo de participación y recordó que durante el período de exposición pública se recibieron 80 alegatos de ayuntamientos, comunidades de montes, asociaciones ecologistas y vecinales, etc., lo que permitió incorporar al catálogo 192 nuevos lugares de especial interés paisajístico que generaron cuatro nuevas áreas de especial interés paisajístico: Pego Negro y San Xusto de Lérez, en la gran área Rías Baixas; Foxo Vello-O Canelar, en la gran área Chairas, Fosas y Serras Ourensanas; y Coto de Codeseda, en la gran área de la Galicia central. Además, se añadieron 28 nuevos miradores, con lo que se pasó de los 444 iniciales a un total de 472. Alberto Núñez Feijóo subrayó que las ventajas que ofrece este catálogo radican en la transparencia con la que fue elaborado ${ }^{6}$, en que proporciona mayor seguridad jurídica, en que fomenta la concienciación en el cuidado del paisaje de Galicia y en que promueve la relevancia del paisaje como recurso de desarrollo económico.

\subsection{El nuevo Plan Territorial de Contingencias por Contaminación Marina Accidental}

El intenso tráfico marítimo, junto con las actividades antrópicas asociadas al litoral de Galicia, supone un riesgo potencial de contaminación de las zonas costeras. En coherencia con ello, la ciudadanía debe contar con la seguridad de que se establecen los mecanismos necesarios para la protección de los bienes con importancia socioeconómica y ambiental ante episodios de contaminación accidental.

\footnotetext{
${ }^{6}$ De momento, y para seguir defendiendo la transparencia, aconsejamos que la página web en la que se dice que está publicada la información funcione. El mensaje que salta al acceder al enlace incluido en el artículo 1 del Decreto 119/2016, de 28 de julio, por el que se aprueba el Catálogo de los Paisajes de Galicia (<http://www.xunta.gal/medio-ambiente-y-ordenacion-del-territorio〉), es: "El recurso solicitado no ha sido encontrado. Está tratando de acceder a http://www.xunta.gal/medio-ambiente-y-ordenacion-delterritorio, actualmente esta página no existe”.
} 
En la actualidad, atendiendo a la vigencia del Plan Territorial de Contaminación Marina Accidental de Galicia (Plan CAMGAL), a la experiencia acumulada en su implementación y al nuevo escenario normativo derivado de la entrada en vigor del Sistema Nacional de Respuesta ante la Contaminación Marina, se hace necesaria la reestructuración de la organización de este plan para reaccionar ante eventuales episodios de contaminación marina accidental en Galicia. Por todo lo expuesto, el 25 de octubre de 2016 se publicó en el DOG el Decreto 135/2016, de 6 de octubre, por el que se regulan la estructura y la organización del Plan Territorial de Contingencias por Contaminación Marina Accidental de la Comunidad Autónoma de Galicia. 